\title{
Estudo do potencial antifúngico e do mecanismo de ação do timol contra cepas de Candida parapsilosis resistentes ao fluconazol e a anfotericina B
}

\author{
Study of the antifungal potential and mechanism of action of thymol against strains of \\ Candida parapsilosis resistant to fluconazole and amphotericin $B$
}

Janiere Pereira de Sousa ${ }^{1}$, Cássio Ilan Soares Medeiros ${ }^{2 *}$, Fillipe de Oliveira Pereira ${ }^{3}$, Felipe Queiroga Sarmento Guerra ${ }^{4}$, Abrahão Alves de Oliveira Filho ${ }^{5}$, Edeltrudes de Oliveira Lima ${ }^{6}$

\begin{abstract}
${ }^{1}$ Doutora em Produtos Naturais e Sintéticos Bioativos pela Universidade Federal da Paraíba (UFPB); ${ }^{2}$ Mestre em Produtos Naturais e Sintéticos Bioativos pela UFPB; ${ }^{3}$ Mestre e Doutor em Produtos Naturais e Sintéticos Bioativos pela UFPB, Professor Adjunto da Universidade Federal de Campina Grande (UFCG); ${ }^{4}$ Mestre e Doutor em Produtos Naturais e Sintéticos Bioativos pela UFPB, Professor Titular da Universidade Federal da Paraiba; ${ }^{5}$ Mestre e Doutor em Produtos Naturais e Sintéticos Bioativos pela UFPB, Professor Adjunto da UFCG; ${ }^{6}$ Mestre e Doutora em Farmácia pela USP, Professora Titular da UFPB, PB
\end{abstract}

\begin{abstract}
Resumo
Objetivo: investigar a suscetibilidade de cepas fúngicas de Candida parapsilosis isoladas de sangue humano frente ao timol, bem como seu mecanismo de ação. Metodologia: foram utilizadas técnicas de microdiluição em placas de 96 poços para determinar a concentração inibitória mínima (CIM) e concentração fungicida mínima (CFM). Além disso, foram realizados testes com o sorbitol e o ergosterol para investigar a ação do timol na parede e na membrana celular fúngica respectivamente. Resultados: nos testes de CIM e CFM, foi observado que as cepas de $C$. parapsilosis são resistentes ao fluconazol e a anfotericina B, no entanto, o timol desempenhou efeito fungicida com razão CFM/CIM entre 1 e 2. Além disso, a CIM do timol não aumentou quando o sorbitol ou o ergosterol foi adicionado no meio, sugerindo fortemente que este monoterpeno não age na parede celular fúngica ou por ligação ao ergosterol na membrana plasmática. Conclusão: portanto, esses resultados contribuem para a elucidação do mecanismo de ação do timol, sugerindo outros possíveis alvos de interação fármaco-receptor. No entanto, mais investigações de caráter enzimático e molecular em modelos in vitro são necessários para que se possa elucidar completamente o modo de ação desse promissor monoterpeno. Palavras-chave: Timol. Candidíase invasiva. Candida parapsilosis. Ação antimicrobiana. Antifúngicos.
\end{abstract}

\begin{abstract}
Objective: to investigate the susceptibility of fungal strains of Candida parapsilosis isolated from human blood against thymol, as well as its mechanism of action. Methodology: microdilution techniques were used in 96-well plates to determine minimum inhibitory concentration (MIC) and minimum fungicidal concentration (MFC). In addition, tests were performed with sorbitol and ergosterol to investigate the action of thymol on the wall and on the fungal cell membrane respectively. Results: in the CIM and CFM tests, it was observed that C. parapsilosis strains are resistant to fluconazole and amphotericin $B$, however, thymol had a fungicidal effect with MFC/MIC ratio between 1 and 2. In addition, thymol MIC did not increase when sorbitol or ergosterol was added in the medium, strongly suggesting that this monoterpene does not act on the fungal cell wall or by binding to ergosterol on the plasma membrane. Conclusion: therefore, these results contribute to the elucidation of the mechanism of action of thymol, suggesting other possible targets of drug-receptor interaction. However, further investigations of enzymatic and molecular character in in vitro models are necessary to fully elucidate the mode of action of this promising monoterpene.

Keywords: Thymol. Invasive candidiasis. Candida parapsilosis. Antimicrobial action. Antifungals.
\end{abstract}

\section{INTRODUÇÃO}

A candidíase é um problema clínico de importância crescente desde a década de 1950, devido à sua frequência e gravidade das suas complicações. Espécies de Candida fazem parte da microbiota normal da pele, boca, trato gastrintestinal e trato geniturinário (FELIX FICH et al., 2014). Entretanto, estes micro-organismos causam significante morbidade e mortalidade em pacientes imu-

Correspondente/Corresponding: *Cássio llan Soares Medeiros - End: Rua Horácio Nóbrega, № 0 , Belo Horizonte, Patos - PB - CEP: 58704 000 - Tel: (83) 3421-3145 - E - mail: cassioism@hotmail.com nocomprometidos. Mais de $70 \%$ das infecções fúngicas invasivas em pacientes hospitalizados são causados por Candida spp., e se apresentam predominantemente como candidemia (ANTINORI et al., 2016).

Embora Candida albicans seja a espécie mais frequentemente associada com candidíase invasiva, espécies não-albicans, como Candida parapsilosis são cada vez mais frequentes (JORDÁN et al., 2014). Isto é preocupante porque a resistência à anfotericina $B$ e ao fluconazol é mais elevada em espécies não-albicans (KEANE et al., 2018). C. parapsilosis também apresenta considerável capacidade de formar biofilmes e, portanto, alta afinidade com dispo- 
sitivos intravasculares e de nutrição parenteral (PRAŻYŃSKA; BOGIEL; GOSPODAREK-KOMKOWSKA, 2018).

Atualmente existem poucos antifúngicos disponíveis para tratar infecções fúngicas invasivas, apenas os polienos, azóis e equinocandinas. Uma desvantagem significativa dos antifúngicos azóis é que geralmente são fungistáticos e seu uso prolongado contribui para o desenvolvimento de resistência (HENDRICKSON et al., 2019). Os polienos apresentam baixos índices de resistência, porém são muito tóxicos. Formulações lipídicas de anfotericina $B$, menos tóxicas que a anfotericina B convencional, e as equinocandinas, nova classe de antifúngicos, apresentam custo muito elevado (DAS; DEVARAJAN, 2020). Assim, a descoberta de novas drogas antifúngicas e novas estratégias terapêuticas é necessária (FREITAS E SILVA et al., 2020).

O timol é um monoterpeno com conhecidas propriedades farmacológicas, incluindo atividade antimicrobiana contra E. coli, S. aureus, (NAJAFLOO et al., 2020) e Candida spp (MARCHESE et al., 2016). Embora estudos anteriores tenham demonstrado o forte potencial antifúngico deste monoterpeno, seu mecanismo de ação ainda é pouco compreendido.

Diante da importância clínica e da alta incidência de candidíase bem como, do aumento do número de indivíduos imunocomprometidos, surgimento de cepas resistentes e toxicidade dos antifúngicos existentes, é de fundamental importância a busca por novos agentes antifúngicos, sendo o timol uma excelente alternativa para esse propósito. Assim, o objetivo desse estudo foi investigar a atividade antifúngica do timol contra cepas de Candida parapsilosis isoladas de sangue humano e seu possível mecanismo de ação.

\section{METODOLOGIA}

\section{Micro-organismos}

Os ensaios foram realizados com sete cepas de $C$. parapsilosis: LM 2, LM 3, LM 4, LM 7, LM 8, LM 9 e LM 14 (isolados clínicos de sangue humano) e uma cepa padrão: C. parapsilosis ATCC 22019. Todas as amostras pertencem à coleção do Laboratório de Microbiologia do Departamento de Farmácia da Universidade Federal do Ceará (UFC), cedidas gentilmente pelo Prof. Dr. Everardo Albuquerque Menezes e Francisco Afrânio Cunha. Todas as cepas foram mantidas em agar sabouraud dextrose (ASD) à $4{ }^{\circ} \mathrm{C}$. Utilizou-se nos ensaios repiques de 24-48 horas incubadas a $35 \pm 2{ }^{\circ} \mathrm{C}$.

\section{Inoculo}

As suspensões foram preparadas a partir de culturas recentes semeadas em ASD e incubadas a $35 \pm 2{ }^{\circ} \mathrm{C}$ durante 24-48 horas. Após a incubação, foi transferido aproximadamente 4-5 colônias (com uma alça estéril) para tubos de ensaio contendo $10 \mathrm{~mL}$ de solução salina estéril ( $\mathrm{NaCl}$ a $0.85 \%$ ). As suspensões resultantes foram agitadas durante 15 segundos com o auxílio de um aparelho vortex (Fanem Ltd., Guarulhos, SP, Brasil). A turbidez do inoculo final foi normalizada utilizando uma suspensão de sulfato de bário (tubo de 0.5 na escala de McFarland). A concentração final obtida foi de 1-5 × $10^{6}$ unidades formadoras de colônias por mililitros (UFC/mL) (CLSI, 2017; SVETAZ et al., 2010).

\section{Substâncias}

As seguintes substâncias utilizadas neste trabalho foram obtidas comercialmente: Timol (2-isopropil-5-metil-fenol) (pureza $\leq 98,5 \%$ ), anfotericina B, fluconazol, sorbitol, ergosterol, RPMI-1640, dimetilsulfóxido (DMSO) e tween 80 (0.02\%) (todos da Sigma-Aldrich, São Paulo, $\mathrm{SP}$, Brasil). O tween 80 e o DMSO foram solubilizados em uma proporção que não excedeu $0.5 \%$ nos testes, posteriormente foi diluído em água destilada estéril com o fitoconstituinte de modo a obter uma emulsão duplamente concentrada de $2048 \mu \mathrm{g} / \mathrm{mL}$ (DE OLIVEIRA PEREIRA et al., 2015).

\section{Testes de Concentração Inibitória Mínima (CIM) e Concentração Fungicida Mínima (MFC)}

A CIM do timol, bem como dos antifúngicos anfotericina $\mathrm{B}$ e fluconazol foram determinados pela técnica da microdiluição em caldo (CLSI, 2017).

Portanto, foram utilizadas placas de 96 orifícios estéreis com tampa. Em cada orifício da placa foram adicionados $100 \mu \mathrm{L}$ do caldo RPMI-1640 duplamente concentrado. Em seguida $100 \mu \mathrm{L}$ da emulsão do produto, também duplamente concentrado foram dispensados nas cavidades da primeira linha da placa. Por meio de uma diluição seriada a uma razão de dois foram obtidas concentrações de $1024 \mu \mathrm{g} / \mathrm{mL}$ até $0,5 \mu \mathrm{g} / \mathrm{mL}$, de modo que na primeira linha da placa se encontra a maior concentração e na última a menor concentração. Por fim, foram adicionados $10 \mu \mathrm{L}$ do inoculo de aproximadamente 1-5 $\times 10^{6} \mathrm{UFC} / \mathrm{mL}$ nas cavidades da placa, onde cada coluna refere-se a uma cepa fúngica especificamente.

Paralelamente, foi realizado controle de viabilidade das cepas ensaiadas $(100 \mu \mathrm{L}$ do mesmo RPMI-1640 duplamente concentrado e $10 \mu \mathrm{L}$ do inoculo de cada cepa). E para verificar a ausência de interferência nos resultados pelos agentes emulsificantes utilizados na solubilização do timol, foi feito um controle no qual foi colocado nas cavidades $100 \mu \mathrm{L}$ do RPMI-1640, DMSO, tween 80 e $10 \mu \mathrm{L}$ da suspensão fúngica. Um controle de esterilidade do meio de cultura também foi realizado, colocando-se $100 \mu \mathrm{L}$ do RPMI-1640 em cavidades sem a suspensão fúngica.

As placas foram assepticamente fechadas e incubadas a $35 \pm 2{ }^{\circ} \mathrm{C}$ por $24-48 \mathrm{~h}$ para a realização da leitura. A CIM foi definida como a menor concentração capaz de inibir o crescimento fúngico visualmente verificado nos orifícios da placa, quando comparado com o crescimento controle. Os ensaios foram realizados em triplicata e a média geométrica foi calculada. De acordo com Svetaz et al. (2010) 
e Pérez Zamora, Torres e Nuñez, (2018), produtos naturais com CIM até $500 \mu \mathrm{g} / \mathrm{mL}$ são considerados com forte poder antimicrobiano; produtos com CIM entre 600 e $1500 \mu \mathrm{g} /$ $\mathrm{mL}$; moderado poder antimicrobiano, e produtos com $\mathrm{CIM}$ acima de $1500 \mu \mathrm{g} / \mathrm{mL}$; fraco poder antimicrobiano.

Após leitura da CIM, alíquotas de $20 \mu \mathrm{L}$ do sobrenadante das cavidades onde foi observada completa inibição do crescimento fúngico nas placas de microdiluição foram semeadas em placas contendo ASD. As placas foram incubadas a $35 \pm 2{ }^{\circ} \mathrm{C}$ por $24-48$ horas. A CFM foi considerada como a menor concentração em que o crescimento microbiano foi inferior a 3 colônias (aproximadamente 99 a 99,5\% de atividade de morte). Os ensaios foram realizados em triplicata e a média geométrica foi calculada (SIDDIQUI et al., 2013).

\section{Ensaio com Sorbitol}

Para investigar a ação do timol na parede celular fúngica foi realizado ensaios com sorbitol, um protetor osmótico usado para estabilizar os protoplastos dos fúngos. A determinação da CIM do timol frente às cepas de C. parapsilosis (4 e ATCC 22019), na presença do sorbitol foram realizados pela técnica de microdiluição conforme descrito anteriormente. Neste caso foi utilizado meio de cultura previamente adicionado de sorbitol $(\mathrm{PM}=182,17)$ a $0,8 \mathrm{M}$. Os valores da CIM do produto foram então comparados na ausência e na presença do sorbitol. Os ensaios foram realizados em triplicata e os resultados expressos pela média geométrica (ESCALANTE et al., 2008; PEIXOTO et al., 2017).

\section{Ensaio com Ergosterol}

Para determinar se o timol interage com o ergosterol das células fúngicas, a CIM do timol contra C. parapsilosis (4 e ATCC 22019) foram determinadas na presença do ergosterol exógeno pelo método de microdiluição conforme descrito anteriormente. Neste caso foi utilizado meio de cultura previamente adicionado de $400 \mu \mathrm{g} / \mathrm{mL}$ de ergosterol. Os valores da CIM do timol foram então comparados na ausência e presença de ergosterol exógeno. Foi realizado o mesmo procedimento com a anfotericina B, cujo mecanismo de ação envolve a interação com ergosterol da membrana celular fúngica, para servir como controle positivo dos resultados. Os ensaios foram realizados em triplicata e os resultados expressos pela média geométrica (PEIXOTO et al., 2017).

\section{Análise Estatística}

Os resultados dos ensaios foram expressos como média \pm erro padrão (e.p.) empregando-se o teste $t$ não pareado para determinar diferenças significantes, quando o valor de $p<0,05$. Os resultados foram analisados com o software Graph Pad Prism versão 6.0 para Windows, San Diego, CA, EUA.

\section{RESULTADOS E DISCURSÃO}

\section{CIM e CFM}

A anfotericina B e o fluconazol estão entre os agentes antifúngicos mais amplamente utilizados no tratamento de infecções fúngicas sistêmicas (FAY et al., 2018; JIA et al., 2019). O fluconazol é amplamente usado na prática clínica devido à sua eficácia e baixa toxicidade. No entanto, com a exposição frequente a este medicamento, isolados de Candida resistentes têm surgido com mais frequência (BERKOW; LOCKHART, 2017; LIU et al., 2020). A necessidade de terapia supressiva com antifúngicos por longo período pode ser responsável pela seleção de cepas resistentes (PÓVOA; COELHO; NORA, 2020; SEYOUM; BITEW; MIHRET, 2020). Esse perfil de resistência antifúngica aos fármacos poliênicos e azóis em comparação com o timol pode ser observado nas tabelas 1 e 2 deste trabalho, o que confirma as premissas expostas anteriormente.

De acordo com o CLSI (2017) cepas com CIM para o fluconazol $\geq 64 \mu \mathrm{g} / \mathrm{mL}$ são consideradas como resistentes a este fármaco, e cepas com CIM para anfotericina $\mathrm{B} \geq$ $2 \mu \mathrm{g} / \mathrm{mL}$ são consideradas resistentes a anfotericina $\mathrm{B}$. Assim, de acordo com essa classificação, neste estudo foi observada resistência ao fluconazol para todas as cepas testadas, e resistência à anfotericina $B$ para 4 cepas de $C$. parapsilosis (Tabela 1).

Tabela 1 - CIM do timol, fluconazol e anfotericina B, e a CFM do timol contra Candida parapsilosis.

\begin{tabular}{lrrccc}
\hline \multirow{2}{*}{ Cepas fúngicas } & \multicolumn{2}{c}{$\begin{array}{c}\text { Timol } \\
(\mu \mathrm{g} / \mathrm{mL})\end{array}$} & $\begin{array}{c}\text { Fluconazol } \\
(\mu \mathrm{g} / \mathrm{mL})\end{array}$ & $\begin{array}{c}\text { Anfotericina } \\
\text { B }(\mu \mathrm{g} / \mathrm{mL})\end{array}$ & $*$ \\
\cline { 2 - 5 } & \multicolumn{1}{c}{ CIM } & \multicolumn{1}{c}{ CFM } & \multicolumn{1}{c}{ CIM } & CIM & \\
\hline LM 2 & 512 & 512 & 128 & 2 & + \\
LM 3 & 512 & 1024 & 128 & 2 & + \\
LM 4 & 256 & 512 & 128 & 2 & + \\
LM 7 & 512 & 1024 & 128 & 2 & + \\
LM 8 & 256 & 512 & $>1024$ & 1 & + \\
LM 9 & 256 & 512 & 1024 & 1 & + \\
LM 14 & 64 & 128 & $>1024$ & 0,5 & + \\
ATCC 22019 & 512 & 1024 & 128 & 1 & + \\
\hline
\end{tabular}

${ }^{*} C$ : controle do crescimento microbiano em RPMI-1640, DMSO (5\%) e tween 80 (2\%), sem timol ou antifúngico.

Fonte: Autoria própria

Tabela 2-CIM ${ }_{50,90}$ e CFM ${ }_{50,90}$ do timol para Candida parapsilosis.

\begin{tabular}{lcccc}
\hline \multirow{2}{*}{ Cepas fúngicas } & \multicolumn{4}{c}{ Timol $(\mu \mathrm{g} / \mathrm{mL})$} \\
\cline { 2 - 5 } & $\mathrm{CIM}_{\mathbf{5 0}}$ & $\mathrm{CIM}_{\mathbf{9 0}}$ & $\mathrm{CFM}_{\mathbf{5 0}}$ & CFM $_{\mathbf{9 0}}$ \\
\hline C. parapsilosis & 256 & 512 & 512 & 1024 \\
\hline
\end{tabular}

Fonte: Autoria própria

A natureza do efeito antifúngico no que diz respeito à inibição do crescimento ou morte dos micro-organismos é importante. A razão entre CFM/CIM para os fungos é usada para especificar a natureza do efeito antimicrobiano 
contra um determinado agente patogênico. Portanto, ficou estabelecido que, quando a razão CFM/CIM de uma molécula antimicrobiana está compreendida entre 1 e 2 , a substância é considerada fungicida contra o agente patogênico. No entanto, se a razão for $>2$, o modo de ação antimicrobiano provavelmente será fungistático (FARSHORI et al., 2010; SIDDIQUI et al., 2013).

De acordo com esses critérios, pode-se observar na tabela 3, que as razões CFM/CIM do timol foram 1 ou 2 para as cepas ensaiadas. Assim, considerando qualquer uma das classificações acima, o timol parece exercer um efeito fungicida contra as cepas testadas. Estes resultados estão de acordo com estudos anteriores, que também constataram a atividade fungicida do timol (CHATRATH et al., 2019; MARCHESE et al., 2016) contra isolados de Candida. A atividade fungicida de um composto é clinicamente mais importante do que a atividade fungistática, pois o uso profilático de fármacos fungistáticos tem sido associado a um aumento da frequência de resistência inata ou adquirida em isolados clínicos de Candida spp. (BHATTACHARYA; SAE-TIA; FRIES, 2020; KSIEZOPOLSKA; GABALDÓN, 2018; REVIE et al., 2018).

Tabela 3-Razão CFM/CIM do timol contra Candida parapsilosis.

\begin{tabular}{lll}
\hline \multirow{2}{*}{ Cepas fúngicas } & \multicolumn{2}{c}{ Timol $(\mu \mathrm{g} / \mathrm{mL})$} \\
\cline { 2 - 3 } & \multicolumn{1}{c}{ CFM/CIM } & \multicolumn{1}{c}{ Efeito } \\
\hline LM 2 & $512 / 512=1$ & Fungicida \\
LM 3 & $1024 / 512=2$ & Fungicida \\
LM 4 & $512 / 256=2$ & Fungicida \\
LM 7 & $1024 / 512=2$ & Fungicida \\
LM 8 & $512 / 256=2$ & Fungicida \\
LM 9 & $512 / 256=2$ & Fungicida \\
LM 14 & $128 / 64=2$ & Fungicida \\
ATCC 22019 & $1024 / 512=2$ & Fungicida \\
\hline
\end{tabular}

Fonte: Autoria própria

Com base nos resultados da CIM e da CFM, uma cepa clínica representativa e a cepa ATCC de $C$. parapsilosis (4 e ATCC 22019) foram escolhidas para os ensaios subsequentes.

\section{Efeitos do Sorbitol}

Duas importantes estruturas fúngicas se apresentam como importantes alvos para a ação de fármacos antifúngicos: a parede celular e a membrana plasmática. Apesar de existir muitos relatos sobre as propriedades antimicrobianas do timol, ainda há poucas investigações sobre o mecanismo de ação antifúngica deste composto em cepas de C. parapsilosis (AHMAD et al., 2011).

A principal classe farmacológica de medicamentos antifúngicos com ação na parede celular dos fungos são as equinocandinas, tais como a micafugina, anidulafungina e caspofungina que inibem a síntese de $\beta$-1,3-glicano, principal constituinte da parede celular, levando a sua desestabilização e morte celular (PATIL; MAJUMDAR, 2017). Portanto, neste estudo foi observado que os valores da CIM do timol contra C. parapsilosis (Tabela 4) não aumentaram na presença do sorbitol $0.8 \mathrm{M}$, sugerindo que estes monoterpenos não atuam através da inibição da síntese da parede celular fúngica, no entanto, provavelmente afetem outro alvo.

Tabela 4 - Efeito do timol contra Candida parapsilosis 4 e ATCC 22019 na ausência e na presença do sorbitol $0.8 \mathrm{M}$.

\begin{tabular}{|c|c|c|c|c|}
\hline \multirow{3}{*}{ Monoterpeno } & \multicolumn{4}{|c|}{ CIM ( $\mu \mathrm{g} / \mathrm{mL})$} \\
\hline & \multicolumn{2}{|c|}{ C. parapsilosis 4} & \multicolumn{2}{|c|}{$\begin{array}{c}\text { C. parapsilosis ATCC } \\
22019\end{array}$} \\
\hline & $\begin{array}{l}\text { Ausência } \\
\text { de sorbitol }\end{array}$ & $\begin{array}{l}\text { Presença } \\
\text { de sorbitol }\end{array}$ & $\begin{array}{l}\text { Ausência } \\
\text { de Sorbitol }\end{array}$ & $\begin{array}{l}\text { Presença } \\
\text { de sorbitol }\end{array}$ \\
\hline Timol & 256 & 256 & 512 & 512 \\
\hline
\end{tabular}

Fonte: Autoria própria

Uma característica distintiva das drogas que atuam inibindo a síntese da parede celular fúngica é que o seu efeito antifúngico é revertido num meio contendo um estabilizador osmótico, tais como o sorbitol (PEIXOTO et al., 2017). Assim, caso o produto atue de alguma forma sob a parede celular, ele causa a lise das células fúngicas na ausência de um estabilizador osmótico, mas permite seu crescimento na presença desse suporte osmótico (ESCALANTE et al., 2008; PRISTOV; GHANNOUM, 2019). E este efeito é detectado por um aumento no valor de CIM em meio com sorbitol em comparação com o valor da CIM no meio sem o sorbitol (meio padrão) (SVETAZ et al., 2010).

Portanto, nesse estudo observou-se que não há nenhuma pesquisa sobre o efeito do timol sobre a parede celular de leveduras do gênero Candida com testes com o sorbitol, dificultando assim a comparação com outros estudos.

\section{Efeitos do Ergosterol}

Existem poucos estudos sobre a interação direta do timol com o ergosterol da membrana celular fúngica. Neste estudo, o monoterpeno testado parece não atuar ao nível de parede celular fúngica. Portanto, outra possibilidade a ser investigada é a possível atuação ao nível de membrana celular. E neste trabalho foi possível observar que o valor da CIM do timol para C. parapsilosis (Tabela 5) permaneceu inalterado na presença de ergosterol $400 \mu \mathrm{g} /$ $\mathrm{mL}$ no meio de cultura, sugerindo que esse monoterpeno não atua por ligação (complexação) com o ergosterol de membrana. Diferentemente do que ocorreu com a anfotericina B (controle positivo) que tem uma conhecida interação com o ergosterol (ESCALANTE et al., 2008; PEIXOTO et al., 2017), que mostrou uma CIM cerca de 100 vezes maior na presença deste esterol. 
Tabela 5 - Efeito do timol e da anfotericina B contra Candida parapsilosis 4 e ATCC 22019 na ausência e na presença de 400 $\mu \mathrm{g} /$ $m L$ do ergosterol.

\begin{tabular}{ccccccc}
\hline & \multicolumn{4}{c}{ CIM $(\mu \mathrm{g} / \mathrm{mL})$} \\
\cline { 2 - 3 } \cline { 5 - 6 } Substância & \multicolumn{2}{c}{ C. parapsilosis 4 } & & C. parapsilosis ATCC 22019 \\
\cline { 2 - 3 } \cline { 6 - 7 } & $\begin{array}{c}\text { Ausência de } \\
\text { ergosterol }\end{array}$ & $\begin{array}{c}\text { Presença de } \\
\text { ergosterol }\end{array}$ & & $\begin{array}{c}\text { Ausência de } \\
\text { ergosterol }\end{array}$ & $\begin{array}{c}\text { Presença de } \\
\text { ergosterol }\end{array}$ \\
Timol & 256 & 256 & & 512 & 512 \\
Anf B & 2 & 256 & & 1 & 128 \\
\hline
\end{tabular}

Fonte: Autoria própria

Além disso, os resultados da interação do timol com o ergosterol deste estudo diferiram dos obtidos por De Lira Mota et al. (2012) com cepas de Rhizopus oryzae, em que o timol interagiu com ergosterol e aumentou o valor da CIM em relação ao controle. Desta forma, há necessidade de mais estudos que venham a esclarecer o mecanismo de ação do timol, pois esse monoterpeno demonstra propriedades antifúngicas significativas e promissoras.

\section{CONCLUSÃO}

Neste estudo, pode-se constatar a atividade antifúngica in vitro de caráter fungicida do timol contra cepas de C. parapsilosis. Efeito este de grande importância para substâncias com essa finalidade, pois os fármacos de caráter fungistático são mais passíveis de induzir resistência antimicrobiana. Além disso, observou-se que o mecanismo de ação do timol provavelmente não envolve a parede celular fúngica diretamente nem a membrana plasmática de $C$. parapsilosis. No entanto, mais investigações são necessárias para que se possa elucidar completamente o modo de ação desse importante monoterpeno.

\section{AGRADECIMENTOS}

Os autores agradecem a Universidade Federal da Paraíba e a CAPES pelo apoio estrutural e financeiro para a realização deste trabalho.

\section{REFERÊNCIAS}

AHMAD, A. et al. Fungicidal activity of thymol and carvacrol by disrupting ergosterol biosynthesis and membrane integrity against Candida. Eur. j. clin. microbiol. infect. dis., Berlin, v. 30, n. 1, p. 41-50, 2011.

ANTINORI, S. et al. Candidemia and invasive candidiasis in adults: A narrative review. Eur j. intern. med., Amsterdam, v. 34, p. 2128, 2016.

BERKOW, E. L.; LOCKHART, S. R. Infection and Drug Resistance Dovepress Fluconazole resistance in Candida species: a current perspective. Infect Drug Resist., [s.I], v. 10, n. 1, p. 237-245, 2017.

BHATTACHARYA, S.; SAE-TIA, S.; FRIES, B. C. Candidiasis and mechanisms of antifungal resistance. Antibiotics, Tokyo, v. 9, n. 6, p. 1-19, 2020.

CHATRATH, A. et al. In vitro anti-biofilm activities of citral and thymol against Candida tropicalis. J Fungi, [s.I], v. 5, n. 1, 2019.

CLINICAL AND LABORATORY STANDARDS INSTITUTE (CLSI). Reference method for broth dilution antifungal susceptibility testing of yeasts. 4th ed. CLSI 2017.
DAS, S.; DEVARAJAN, P. V. Enhancing Safety and efficacy by altering the toxic aggregated state of amphotericin $b$ in lipidic nanoformulations. Mol. pharm., Washingtom,v. 17, n. 6, p. 2186-2195, 2020.

DE LIRA MOTA, K. S. et al. Antifungal activity of thymus vulgaris I. essential oil and its constituent phytochemicals against rhizopus oryzae: Interaction with ergosterol. Molecules, Basel, v. 17, n. 12, p. 14418-14433, 2012.

DE OLIVEIRA PEREIRA, F. et al. Antifungal activity of geraniol and citronellol, two monoterpenes alcohols, against Trichophyton rubrum involves inhibition of ergosterol biosynthesis. Pharm. Biol., Lisse, v. 53, n. 2, p. 228-234, 2015.

ESCALANTE, A. et al. Evidence for the mechanism of action of the antifungal phytolaccoside $B$ isolated from Phytolacca tetramera Hauman. J. nat. prod., Cincinnati, v. 71, n. 10, p. 1720-1725, 2008.

FARSHORI, N. N. et al. Synthesis, characterization, and in vitro antimicrobial activities of 5-alkenyl/hydroxyalkenyl-2-phenylamine1,3,4-oxadiazoles and thiadiazoles. Bioorg med. chem lett., Oxford, v. 20, n. 6, p. 1933-1938, 2010.

FAY, V. DA S. et al. Drug susceptibility in emerging fungal infections: Tests with fluconazole, itraconazole, and amphotericin B. An. bras. dermatol., Riode jJaneiro, v. 93, n. 3, p. 462-464, 2018.

$\mathrm{FICH}$, F. et al. Candida Parapsilosis and candida guillermondii: emerging pathogens in nail candidiasis. Ind. j. dermatol., v. 1, n. 59, p. 24-29, 2014.

FREITAS E SILVA, K. S. et al. Setting new routes for antifungal drug discovery against pathogenic fungi. Curr. pharm. des., Schipol, v. 26, n. 14 , p. 1509-1520, 2020.

HENDRICKSON, J. A. et al. Antifungal Resistance: a Concerning Trend for the Present and Future. Curr. Infect. Dis. Rep., [s.I], v. 21, n. 12, 2019.

JIA, C. et al. Synergistic effects of geldanamycin with fluconazole are associated with reactive oxygen species in Candida tropicalis resistant to azoles and amphotericin B. Free rad. res., Yverdon, v. 53, n. 6, p. 618-628, 2019

JORDÁN, I. et al. C. albicans, C. parapsilosis and C. tropicalis invasive infections in the PICU: clinical features, prognosis and mortality. Rev. esp. quimioter., Barcelona, v. 27, n. 1, p. 56-62, 2014.

KEANE, S. et al. Systematic review on the first line treatment of amphotericin B in critically ill adults with candidemia or invasive candidiasis. Expert Ver. Anti-Infect Ther., [s.I], v. 16, n. 11, p. 839-847, 2018.

KSIEZOPOLSKA, E.; GABALDÓN, T. Evolutionary emergence of drug resistance in candida opportunistic pathogens. Genes, Oxford, v. 9, n. 9, 2018.

LIU, Y. et al. The synergistic antifungal effects of gypenosides combined with fluconazole against resistant Candida albicans via inhibiting the drug efflux and biofilm formation. Biomed. pharmacother., Paris, v. 130, n. June, p. 110580, 2020.

MARCHESE, A. et al. Antibacterial and antifungal activities of thymol: A brief review of the literature. Food Cchem., Barking,v. 210, p. 402414, 2016.

NAJAFLOO, R. et al. A mini-review of Thymol incorporated materials: Applications in antibacterial wound dressing. J. drug deliv. sci. technol., Paris, v. 60, p. 101904, 2020.

PATIL, A.; MAJUMDAR, S. Echinocandins in antifungal pharmacotherapy. J. pharm pharmacol., London, v. 69, n. 12, p. 1635-1660, 2017.

PEIXOTO, L. R. et al. Antifungal activity, mode of action and anti-biofilm effects of Laurus nobilis Linnaeus essential oil against Candida spp. Arch. 
oral biol .,Oxford, v. 73, p. 179-185, 2017.

PÉREZ ZAMORA, C. M.; TORRES, C. A.; NUÑEZ, M. B. Antimicrobial activity and chemical composition of essential oils from Verbenaceae species growing in South America. Molecules, Basel, v. 23, n. 3, 2018.

PÓVOA, P. et al. Antifungal use in the surgical ICU patient. Curr. opin. anaesthesiol. Philadelphia, v. 33, n. 2, p. 131-138, 2020.

PRAŻYŃSKA, M.; BOGIEL, T.; GOSPODAREK-KOMKOWSKA, E. In vitro activity of micafungin against biofilms of Candida albicans, Candida glabrata, and Candida parapsilosis at different stages of maturation. Folia Microbiol.,mPraha, v. 63, n. 2, p. 209-216, 2018.

PRISTOV, K. E.; GHANNOUM, M. A. Resistance of Candida to azoles and echinocandins worldwide. Clin. microbiol. infect., Oxford, v. 25, n. 7, p. 792-798, 2019.
REVIE, N. M. et al. Antifungal drug resistance: evolution, mechanisms and impact. Curr. opin. microbiol., New York, v. 45, n.2, p. 70--76, 2018.

SEYOUM, E.; BITEW, A.; MIHRET, A. Distribution of Candida albicans and non-albicans Candida species isolated in different clinical samples and their in vitro antifungal suscetibity profile in Ethiopia. BMC infect. dis., London, v. 20, n. 1, p. 1-9, 2020.

SIDDIQUI, Z. N. et al. Synthesis, characterization and antimicrobial evaluation of novel halopyrazole derivatives. J Saudi Chem. Soc., [s.I], v. 17, n. 2, p. 237-243, 2013.

SVETAZ, L. et al. Value of the ethnomedical information for the discovery of plants with antifungal properties. A survey among seven Latin American countries. J. ethnopharmacol., Limerick, v. 127, n. 1, p. 137-158, 2010.

Submetido em: 06/08/2019

Aceito em:26/10/2020 